\section{Influence of channel BER on IEEE 802.11 DCF}

\section{P. Chatzimisios, A.C. Boucouvalas and V. Vitsas}

A new performance model of the IEEE 802.11 protocol is introduced that calculates throughput, average packet delay, packet drop probability and average packet drop time. This model is validated with simulation results using the OPNET $^{\mathrm{TM}}$ simulation package. The proposed model predicts 802.11 protocol performance very accurately since it considers both transmission errors and packet retry limits. The effect of errors and network size on the performance of the basic access scheme, in terms of throughput, packet delay, packet drop time and drop probability is explored.

Introduction: The IEEE 802.11 protocol [1] is the dominating standard for Wireless Local Area Networks (WLANs) and employs distributed co-ordination function (DCF) as the essential medium access control method. In DCF, a station transmits if the medium is idle. If the medium is sensed busy, the station waits until the current transmission is over. The station then defers for a randomly selected time interval to minimise collisions before transmitting. Each station maintains a retry count that indicates the number of retransmission attempts of a data packet. If the retry count reaches the specified limit, the packet is dropped.

Since the release of the IEEE 802.11 standard many research efforts have been conducted on modelling IEEE 802.11 DCF. Bianchi in [2] uses a Markov chain to model the idealistic assumption that packet retransmissions are unlimited and a packet is being transmitted continuously until its successful reception. Wu et al. in [3] extends Bianchi's analysis to include the finite packet retry limits as defined in the IEEE 802.11 standard.

In this Letter we introduce a mathematical model which extends the approach in [3] by taking into account both channel bit errors and packet retry limits for the basic access scheme. Our new performance model allows the calculation of the throughput efficiency, the average packet delay, the packet drop probability and the average time to drop a packet for the IEEE 802.11 protocol. Using OPNET simulation results, we validate our mathematical model and show that our model predicts DCF performance more accurately than Bianchi's model [2] that does not consider packet retry limits. Analytical results utilising our proposed model study the dependence of the protocol performance on the bit error rate (BER) and the network size.

Analysis: Our analysis assumes that the network consists of $n$ contending stations and that each station has always a packet ready for transmission. The key assumption of our model is that the collision-error probability $p$ of a transmitted packet is constant and independent of the number of collisions or errors this packet has suffered in the past.

Before initiating a packet transmission, each station's backoff timer is uniformly chosen in the interval $\left[0, W_{i}-1\right]$ where $W_{i}$ is the current contention window size and $i$ is the backoff stage. The value of $W_{i}$ depends on the number of failed transmissions of the packet; at the first transmission attempt, $W_{0}=W$. After each retransmission due to a packet collision or error, $W_{i}$ is doubled up to a maximum value, $W_{m^{\prime}}=W \cdot 2^{m^{\prime}}$ where $m^{\prime}$ is the number of backoff stages.

The discrete-time Markov chain of our model is not shown in this Letter due to limited space as is the case with the Markov chain utilised in [3]. Since our analysis considers transmission errors, $p$ is now the probability that a transmitted packet encounters a collision or is received in error and is given by:

$$
p=1-(1-\tau)^{n-1} \cdot(1-\mathrm{BER})^{l+H}
$$

where BER is the link bit error rate, $l$ is the packet size, $H$ is the packet header length and $\tau$ is the probability that a station transmits a packet in a randomly chosen slot time. Using the Markov chain $\tau$ is calculated as:

$$
\begin{aligned}
& \tau=\frac{2 \cdot(1-2 p) \cdot\left(1-p^{m+1}\right)}{W \cdot\left(1-(2 p)^{m^{\prime}+1}\right) \cdot(1-p)+(1-2 p) \cdot\left[W \cdot 2^{m^{\prime}}\right.} \\
& \left.\times p^{m^{\prime}+1} \cdot\left(1-p^{m-m^{\prime}}\right)+1-p^{m+1}\right]
\end{aligned}
$$

Equations (1) and (2) form a nonlinear system with two unknowns $p$ and $\tau$. This nonlinear system can be solved using numerical methods and has a unique solution.

Let $P_{t r}$ be the conditional probability that at least one transmission occurs in a randomly chosen slot time, $P_{s}$ the conditional probability that this transmission is successful and PER the packet error rate, therefore:

$$
P_{t r}=1-(1-\tau)^{n}, \quad P_{s}=\frac{n \cdot \tau \cdot(1-\tau)^{n-1}}{1-(1-\tau)^{n}} \cdot(1-\mathrm{PER})
$$

The probability $P_{c}$ that an occurring transmission collides and the probability $P_{e r}$ that a packet is received in error are given by:

$$
P_{c}=1-\frac{n \cdot \tau \cdot(1-\tau)^{n-1}}{1-(1-\tau)^{n}}, \quad P_{e r}=\frac{n \cdot \tau \cdot(1-\tau)^{n-1}}{1-(1-\tau)^{n}} \cdot \text { PER }
$$

The saturation throughput $S$ is given by:

$$
\begin{aligned}
S & =\frac{P_{t r} \cdot P_{s} \cdot l}{E[\text { slot }]} \\
& =\frac{P_{t r} \cdot P_{s} \cdot l}{\left(1-P_{t r}\right) \cdot \sigma+P_{t r} \cdot P_{s} \cdot T_{s}+P_{t r} \cdot P_{c} \cdot T_{c}+P_{t r} \cdot P_{e r} \cdot T_{e r}}
\end{aligned}
$$

where $E[\mathrm{slot}]$ is the average length of a slot time, $\sigma$ is the duration of an empty slot, $T_{s}, T_{c}$ and $T_{e r}$ are the average time intervals that the medium is sensed busy due to a successful transmission, a collision or an error transmission, respectively.

The packet drop probability is defined as the probability that a packet is dropped when the retry limit is reached and is equal to:

$$
p_{\text {drop }}=p^{m+1}
$$

Since the average number of slot times a station defers in the $i$ stage is $d_{i}=\left(W_{i}+1\right) / 2$, the average time to drop a packet is given by:

$$
\begin{aligned}
E\left[D_{\text {drop }}\right] & =\sum_{i=0}^{m} \frac{W_{i}+1}{2} \cdot E[\text { slot }] \\
& =\frac{W \cdot\left(2^{m^{\prime}+1}-1\right)+W \cdot 2^{m^{\prime}} \cdot\left(m-m^{\prime}\right)+(m+1)}{2} \cdot E[\text { slot }]
\end{aligned}
$$

The average packet delay $E[D]$ for a successfully transmitted packet is given by:

$$
E[D]=E[X] \cdot E[\text { slot }]=\sum_{i=0}^{m}\left[\frac{W_{i}+1}{2} \cdot \frac{\left(p^{i}-p^{m+1}\right)}{1-p^{m+1}}\right] \cdot E[\text { slot }]
$$

where $E[X]$ is the average number of slot times required for a successful packet transmission and is calculated by multiplying the average number of slot times $d_{i}$ the packet is delayed in each backoff stage by the probability of a packet which is not dropped, reaches the $i$ stage.

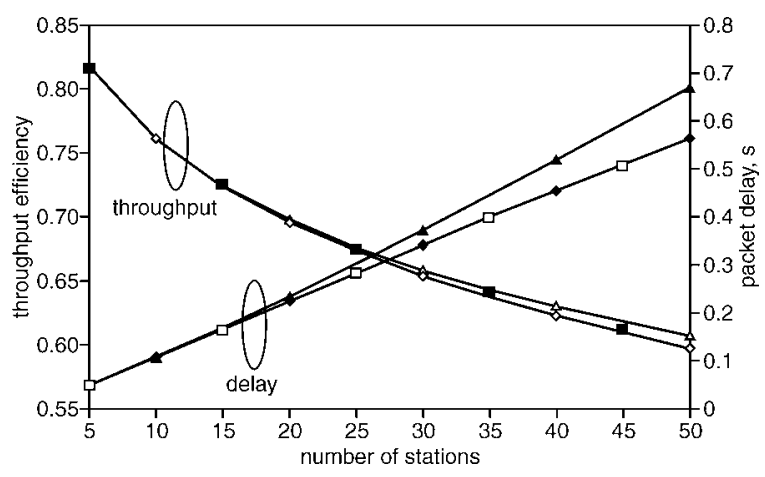

Fig. 1 Throughput efficiency and packet delay against $n$, for $W=32$, $l=8184$ bits, $m^{\prime}=5, C=1 \mathrm{Mbit} / \mathrm{s}, \mathrm{BER}=0$

$\triangle$ throughput efficiency, no retry limits

- throughput efficiency, OPNET simulation

throughput efficiency, $m=6 \quad \Delta$ packet delay, no retry limits

$\square$ packet delay, OPNET simulation $\bullet$ packet delay, $m=6$

Results: Fig. 1 shows throughput efficiency and packet delay against the number of contending stations for an error-free channel. Results obtained from our analytical model are compared to simulation 
outcome and to results obtained using the model in [2] that does not use packet retry limits. Fig. 1 validates our analytical model since an almost exact match is observed between analytical results (lines) and simulation outcome (symbols) obtained by means of our IEEE 802.11 simulator developed with the OPNET ${ }^{\mathrm{TM}}$ simulation package. Moreover, the Figure illustrates that analytical modelling that considers retry limits predicts very accurately DCF throughput performance, a conclusion not drawn in [3] which added retry limits in the analytical model in [2].

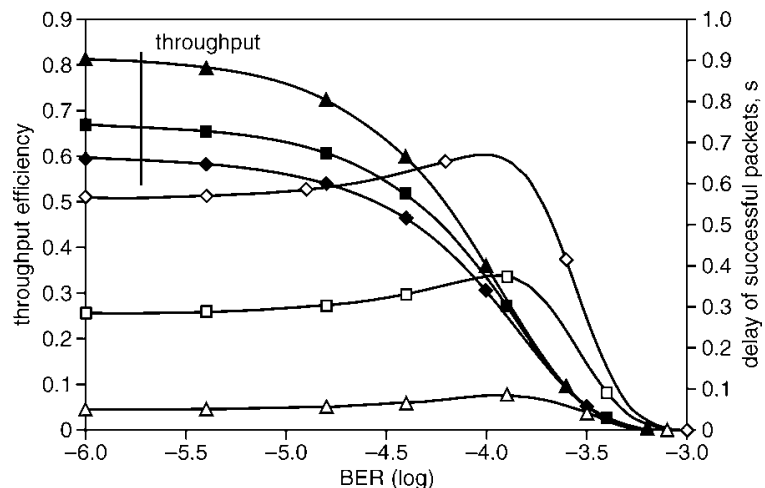

Fig. 2 Throughput efficiency and packet delay against BER, for $W=32$, $l=8184 \mathrm{bits}, \mathrm{m}^{\prime}=5, \mathrm{C}=1 \mathrm{Mbit} / \mathrm{s}$

$\Delta$ throughput efficiency, $n=5$

throughput efficiency, $n=50$

$\square$ packet delay, $n=25$

throughput efficiency, $n=25$

$\triangle$ packet delay, $n=5$

$\diamond$ packet delay, $n=50$

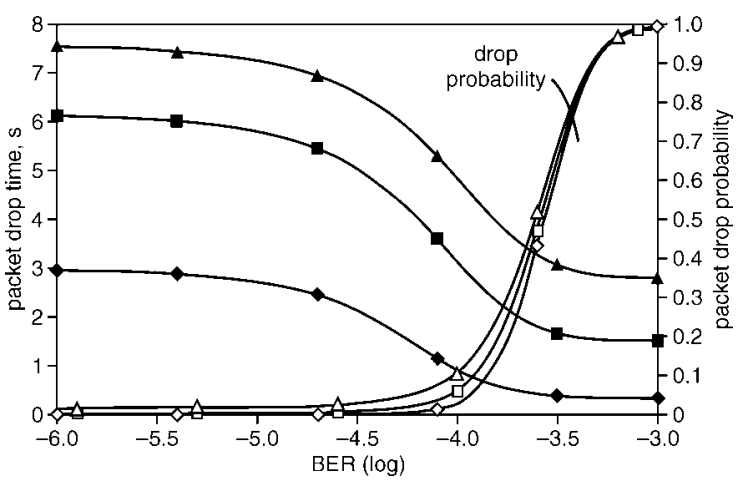

Fig. 3 Packet drop time and packet drop probability against BER, for $W=32, l=8184$ bits, $m^{\prime}=5, C=1 \mathrm{Mbit} / \mathrm{s}$

- packet drop time, $n=5$

$\Delta$ packet drop time, $n=50$

$\square$ packet drop probability, $n=25$

- packet drop time, $n=25$

$\diamond$ packet drop probability, $n=5$

$\triangle$ packet drop probability, $n=50$

Figs. 2 and 3 study the effect of transmission errors by plotting throughput efficiency, average packet delay, average packet drop time and packet drop probability for three representative network sizes $(n=5,25$ and 50). Fig. 2 shows that when BER increases, throughput always degrades; packet delay increases, reaches a maximum value and then decreases gradually and finally drops to 0 . When BER is high enough to result in a significant increase in the drop probability (as shown in Fig. 3), packet delay starts decreasing. Thus, the low packet delay values at high BER concern only a small number of successfully received packets due to high drop probability and, therefore, have a very small significance. The maximum in the average packet delay curve (Fig. 2) can be explained as follows. At low BER, increasing BER results in packet delay growth due to an increased number of packet retransmissions which highly delay the successful packet reception. In this case, high backoff stages are rarely used. If BER becomes high enough to result in an increased number of dropped packets, the average packet delay decreases for the following two reasons: (i) the long delays of the dropped packets do not contribute to the average packet delay and (ii) at high BER, high backoff stages with large contention window sizes are more often used. Thus, successfully transmitted packets are less delayed by transmissions of other stations that utilise high contention window sizes.

Fig. 3 illustrates that packet drop probability increases rapidly when $\operatorname{BER}>10^{-4}$ due to the increased number of packet error transmissions. Conversely, network size only marginally affects packet drop probability. Fig. 3 also shows that packet drop time is highly sensitive to the number of contending stations. Moreover, increasing the BER results in a decrease in the packet drop time regardless of the network size. The level of decrease of the packet drop time increases with increasing BER, but when the packet drop probability starts increasing rapidly, the packet drop time stays at a constant level.

Conclusions: A new mathematical model is introduced that considers both packet retry limits and transmission errors in order to calculate the performance of the IEEE 802.11 protocol. Analytical results show that protocol performance strongly depends on the bit error rate. Increasing BER results in decreasing throughput and increasing packet drop probability. The delay of successfully received packets shows a different behaviour when BER increases; it starts increasing and, after reaching a maximum value, it decreases gradually and finally drops to 0 . Moreover, performance is highly dependent on the network size; increasing network size results in a decrease in throughput but an increase in packet drop time and packet delay.

\section{(C) IEE 2003}

Electronics Letters Online No: 20031081 DOI: 10.1049/el:20031081

26 August 2003

P. Chatzimisios and A.C. Boucouvalas (Design, Engineering and Computing, Multimedia Communications Research Group, Bournemouth University, Fern Barrow, Poole, Dorset BH12 5BB, United Kingdom)

V. Vitsas (Department of Information Technology, Technological Educational Institution, Thessaloniki, Greece)

\section{References}

1 'IEEE standard for Wireless LAN Medium Access Control (MAC) and Physical Layer (PHY) Specification: High-Speed Physical Layer Extension in the 2.4 GHz Band, IEEE 802.11b WG, September 1999

2 BIANCHI, G.: 'Performance analysis of the IEEE 802.11 distributed coordination function', IEEE J. Sel. Areas Commun., 2000, 18, (3), pp. $535-547$

3 wU, H., et al.: 'Performance of Reliable Transport Protocol over IEEE 802.11 Wireless LAN: Analysis and Enhancement'. Proc. IEEE INFOCOM 2002, 2002, Vol. 2, pp. 599-607 